\title{
HE0241-0155 - Evidence for a large scale homogeneous field in a highly magnetic white dwarf ${ }^{\star}$
}

\author{
D. Reimers ${ }^{1}$, S. Jordan ${ }^{2}$, and N. Christlieb ${ }^{1}$ \\ 1 Hamburger Sternwarte, Universität Hamburg, Gojenbergsweg 112, 21029 Hamburg, Germany \\ 2 Institut für Astronomie und Astrophysik, Eberhard-Karls-Universität Tübingen, Sand 1, 72076 Tübingen, Germany
}

Received 10 Septembre 2003 / Accepted 3 November 2003

\begin{abstract}
In the course of the Hamburg/ESO survey we have discovered a white dwarf whose spectrum exhibits many similarities with the prototype of magnetic white dwarfs Grw $+70^{\circ} 8247$. In particular several stationary line components indicative for magnetic fields between about 150 and $400 \mathrm{MG}$ are found in both objects. However, the features between 5000 and $5500 \AA$ in the spectrum of HE0241-0155 cannot be explained by stationary line components and demand a relatively homogeneous magnetic field with clustering around $200 \mathrm{MG}$. For this reason a pure dipole model failed to reproduce this spectral region. An offset-dipole configuration led to some improvement in the fit but a good agreement was only possible for a geometry described by an expansion into spherical harmonics - where most of the surface is covered with magnetic field strengths strongly clustered around $200 \mathrm{MG}$. This may indicate the presence of a large magnetic spot whose presence could be tested with time resolved spectro-polarimetry.
\end{abstract}

Key words. stars: white dwarfs - stars: magnetic fields - radiative transfer - magnetic fields

\section{Introduction}

A few percent (5\%) of all known white dwarfs have detectable magnetic fields, and the true fraction may be as high as $10 \%$ if selection effects are taken into account (Harris et al. 2003). However, the number of white dwarfs with field strengths above $100 \mathrm{MG}$, where the spectral line pattern is no longer similar to normal $\mathrm{H}$ or $\mathrm{He}$ I spectra, is still small, and each star is unique. Most of these stars have been detected serendipitously during follow-up spectroscopy by surveys designed for other purposes, e.g. quasar surveys like the Palomar-Green Survey (Schmidt \& Green 1983) or the Hamburg/ESO survey (Wisotzki et al. 1996; Reimers \& Wisotzki 1997). The first star of this type was Grw $+70^{\circ} 8247$, whose unique spectrum was first observed by by Minkowski (1938). Its shallow spectral features resisted a physical identification for decades. Kemp (1970), following an idea by J. Landstreet, proposed that a magnetic field would produce circular dichroism, and discovered circular dichroism in Grw $+70^{\circ} 8247$. Only progress in quantum mechanical calculations of the hydrogen energy levels in strong magnetic fields made possible an identification (Praddande 1972; Rösner et al. 1984). Details of the Grw $+70^{\circ} 8247$ spectrum and its interpretation are given by Greenstein (1984), Angel et al. (1985) and Wickramasinghe \& Ferrario (1988). In this letter we report on the serendipitous

Send offprint requests to: D. Reimers, e-mail: dreimers@hs.uni-hamburg.de

* Based on observations collected at the European Southern Observatory, La Silla, Chile.
Table 1. Journal of observations.

\begin{tabular}{llrr}
\hline \hline Date & Tel./instrument & Resolution & Exp. time \\
\hline $13 / 9 / 98$ & $1.52 /$ B \& C & $15 \AA$ & $8 \mathrm{~min}$ \\
$28 / 11 / 98$ & $1.54 /$ DFOSC & $5 \AA$ & $30 \mathrm{~min}$ \\
\hline
\end{tabular}

discovery of a near "twin" to Grw $+70^{\circ} 8247$ in the course of the Hamburg/ESO survey. We give a physical interpretation of its spectra using Jordan's code (Jordan 1988, 1992; Euchner et al. 2002) in terms of de-centered magnetic dipole fields and we briefly discuss the remaining discrepancies between observations and theory.

\section{Observations}

HE 0251-0155 has been discovered as a quasar candidate within the Hamburg/ESO (HE) Survey (Wisotzki et al. 1996; Reimers \& Wisotzki 1997). The journal of spectroscopic observations with ESO telescopes is given in Table 1. Wavelength and flux calibration followed standard procedures. From the two available spectra (shown in Fig. 1) there is no evidence for variability so far. Coordinates are RA $2^{\mathrm{h}} 44^{\mathrm{m}} 22^{\mathrm{s}} .0$ Dec $-01^{\circ} 42^{\prime} 41^{\prime \prime}$ (equinox 2000.0, epoche 1982.783), brightness is $B=16.7$.

Prominent broad absorption lines are seen at 4060, 4170, 4280, 4435, 4690, 5093, 5220, 5390, and $5830 \AA$. Further lines in the red are at 6780,7310 and $8300 \AA$ (Fig. 1). Note, however, that the features in the red are partly contaminated 


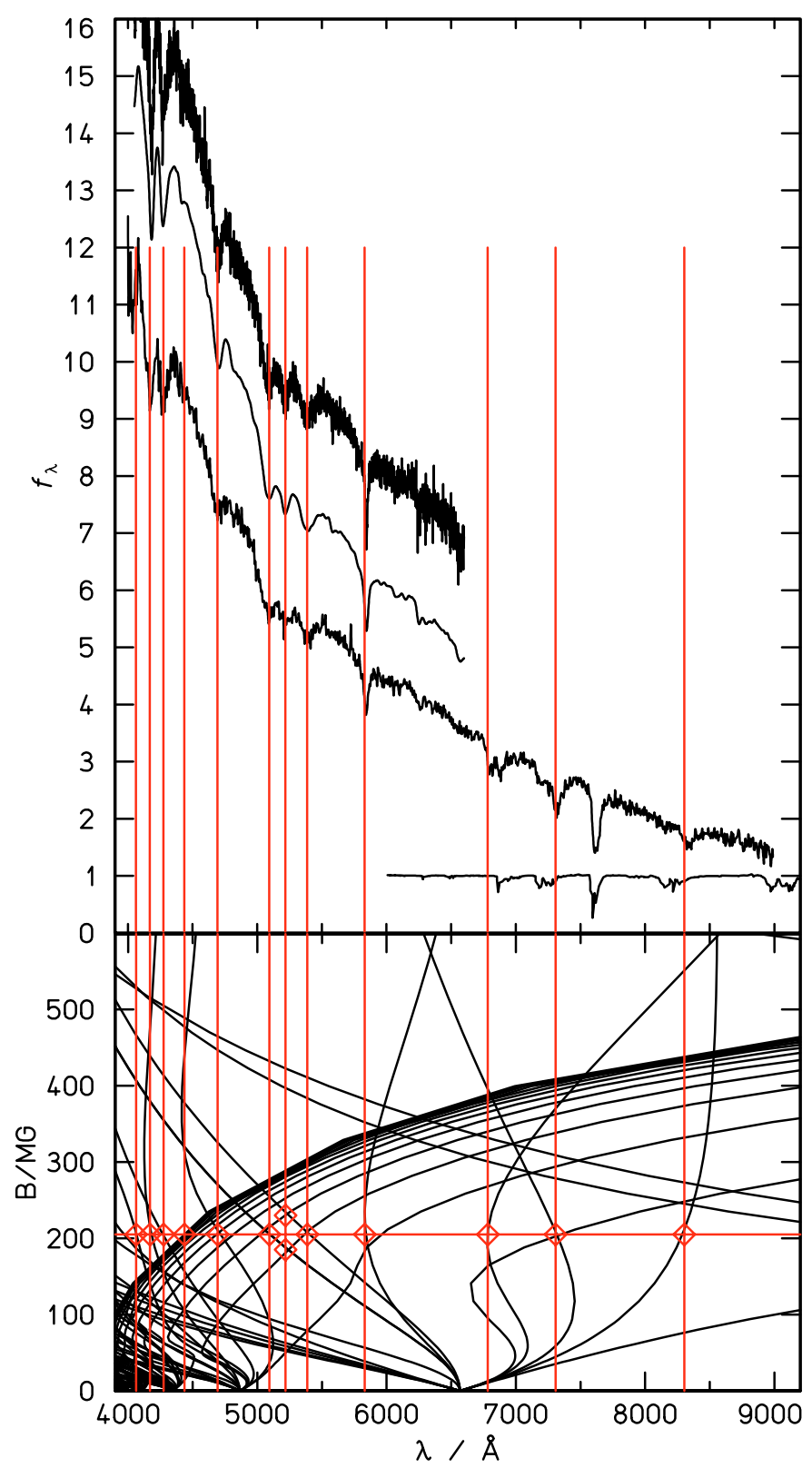

Fig. 1. Observed spectra of HE0241-0155 compared to the position of the hydrogen line components. The upper spectrum (obtained 28/11/98) with a resolution of $5 \AA$ has been smoothed (second curve) in order to match the $15 \AA$ resolution of the lower spectrum (13/9/98). The main features arise from magnetic field strengths of about $200 \mathrm{MG}$ (marked with circles) and are not in all cases due to stationary line components. In order to identify the telluric features a spectrum of HD7041 by Stevenson (1994) observed at an airmass 1.7 is shown.

by telluric features, which can be identified by the comparison spectrum of HD7041 taken by Stevenson (1994) at high airmass, also shown in Fig. 1. A comparison with known magnetic white dwarfs shows its obvious similarity with Grw $+70^{\circ} 8247$ which is a DA with a 320 MG dipole field according to Wickramasinghe \& Ferrario (1988) and Jordan (1989).

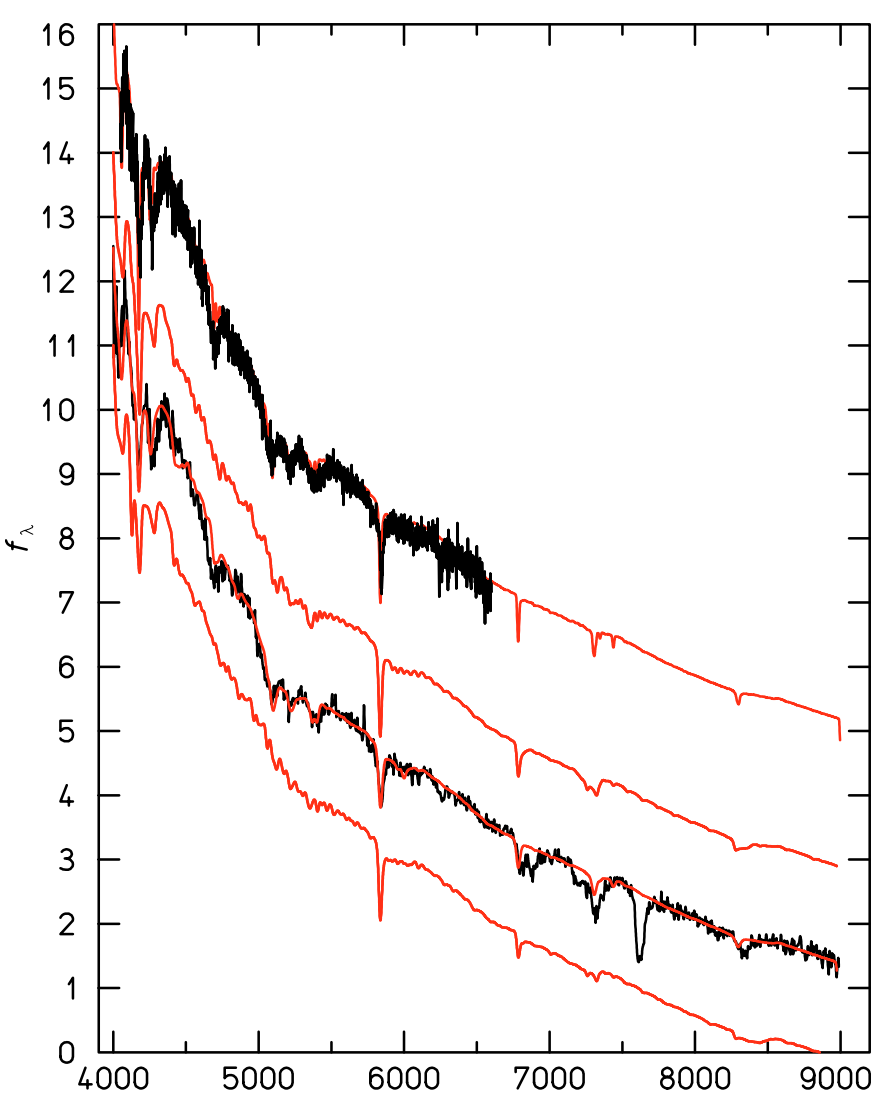

Fig. 2. Best fit for a magnetic field geometry described by an expansion into spherical harmonics up to $l=4$ with $m=0$ (convolved with a $5 \AA$ Gaussian and plotted over the low resolution spectrum, second curve from below), a pure dipole field (lower curve), and a dipole with an offset along the magnetic field axis (second curve from above). For comparison the upper curves show the high resolution spectrum together with the best fit model convolved with a Gaussian of $5 \AA$.

\section{Interpretation and discussion}

The efficiency in modeling the spectra and polarization data of magnetic white dwarfs has considerably improved due to two factors: firstly, instead of solving the radiative transfer equations on thousands of surface elements on the star for each model, databases for flux and polarization spectra computed for a wide range of field strengths, viewing angles, and effective temperatures are used. Secondly, either evolutionary strategies or genetic algorithms, and a subsequent downhill simplex algorithm (Press et al. 1992) are used in order to find the best fit to the observations. The approach used in this paper is very similar to the one described by Euchner et al. (2002) with the exceptions that a genetic algorithm (Caroll 2001) has been used, more viewing angles (12 instead of 9), and an interpolation (12 $\mu$ angles) instead of a linear approximation are used.

If a pure magnetic dipole is assumed, the best fit (Fig. 2) to the observed data was obtained for a polar field strength of $398 \mathrm{MG}$, with the dipole axis oriented almost $\left(i=-86^{\circ}\right)$ perpendicular to the observer, and an effective temperature of $12000 \mathrm{~K}$. However, this model fails to account for the features at 5093, 5218, and $5287 \AA$. These features do not correspond to any stationary line component in any reasonable range of magnetic field strengths; the closest agreement would 


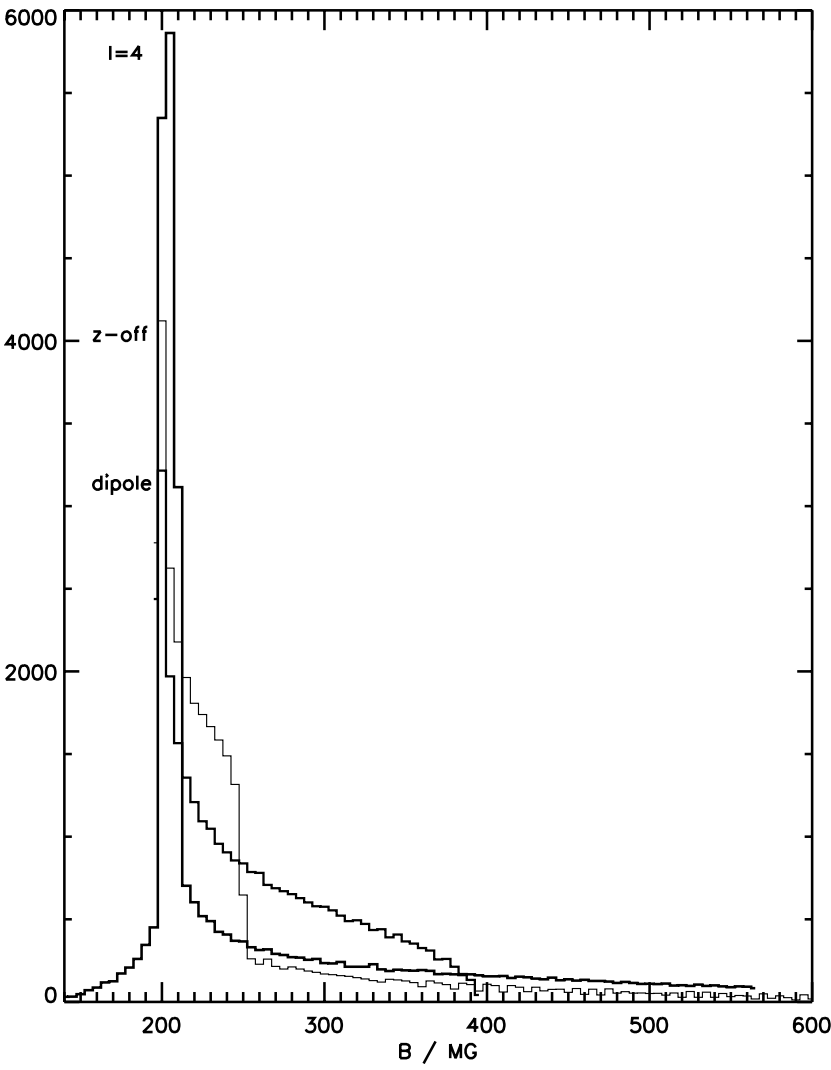

Fig. 3. Distribution of the absolute values of the magnetic field strength over the observed hemisphere predicted by the three best fit models: the dipole model (thick line), the off-set dipole model (medium), and expansion into spherical harmonics (thin) up to $l=4$ $(m=0)$.

have the $4 \mathrm{f}_{-1} \rightarrow 2 \mathrm{~s} 0$ components with a maximum at $5119 \AA$ and $61 \mathrm{MG}$. The wavelengths of stationary line components go through maxima or minima as functions of the magnetic field strength; they are visible in the spectra of magnetic white dwarfs despite a considerable variation of the field strengths as in the case of a pure magnetic dipole (where the field strength is a factor of two higher at the pole than at the equator, and the distribution of the magnetic field strengths over the visible stellar surface is relatively flat (see Fig. 3).

As indicated by the circles in Fig 1, the features missing in the fit could be due to a relatively homogeneous field of about $200 \mathrm{MG}$. One possibility to describe a field distribution where the majority of the surface is covered by a magnetic field close to $200 \mathrm{MG}$ is a dipole field with a large offset of the dipole relative to the center of the star. Although the total variation of field strengths is larger in this case, more magnetic field strengths cluster around one relatively homogeneous magnetic field on one pole.

The best fit for an offset dipole (only offsets along the magnetic axis are considered) is obtained for the same polar field strength (399 MG) and the same orientation $\left(i=-86^{\circ}\right)$, but for an offset of 0.16 stellar radii away from the observer. The histogram Fig. 3 shows the effect: the magnetic field strengths are now even more strongly peaked at $200 \mathrm{MG}$ and the vast majority of surface elements has a magnetic field between 200 and
250 MG. The resulting fit (upper curve of Fig. 2) has improved considerably, and particularly the missing three line features are now visible - although still somewhat too shallow - in the theoretical model.

Finally, a more general approach, an expansion of the magnetic field into spherical harmonics, assuming that the source of the magnetic field lie entirely within the star (Jacobs 1987):

$$
\begin{aligned}
& B_{r}=-\sum_{l=1}^{\infty} \sum_{m=0}^{l}(l+1)\left(g_{l}^{m} \cos m \phi+h_{l}^{m} \sin m \phi\right) P_{l}^{m}(\cos \theta) \\
& B_{\theta}=+\sum_{l=1}^{\infty} \sum_{m=0}^{l}\left(g_{l}^{m} \cos m \phi+h_{l}^{m} \sin m \phi\right) \mathrm{d} P_{l}^{m}(\cos \theta) / \mathrm{d} \theta \\
& B_{\phi}=-\sum_{l=1}^{\infty} \sum_{m=0}^{l} m\left(g_{l}^{m} \cos m \phi+h_{l}^{m} \sin m \phi\right) \mathrm{d} P_{l}^{m}(\cos \theta) / \mathrm{d} \sin \theta
\end{aligned}
$$

with the associated Legendre polynomials $P_{l}^{m}$. The components are given in spherical coordinates $r, \theta$, and $\phi$. For this paper we limited ourselves to $l \leq 4$ and $m=0$, leading to 4 free parameters beside the orientation of the magnetic axis relative to the observer. Any attempt to account for the $20 m \neq 0$ parameters, did only slightly improve the solution. Since no polarization data additionally constrain the distribution of the longitudinal component of the magnetic field, we present only the best fit for $m=0: g_{1}^{0}=172 \mathrm{MG}, g_{2}^{0}=74 \mathrm{MG}, g_{3}^{0}=0.3 \mathrm{MG}$, $g_{4}^{0}=-0.1 \mathrm{MG}$, and $i=-97^{\circ}$.

Figure 2 shows now that the overall agreement between observation and theoretical fit is almost perfect. The corresponding histogram is now even stronger peaked around $200 \mathrm{MG}$.

It is clear that the limitation to spectra without polarization data cannot lead to a unique solution. All distributions that cluster around $200 \mathrm{MG}$ should lead to similar results. Such a strong clustering could be a hint for a magnetic spot with a relative homogeneous magnetic field covering a large portion of the visible hemisphere, as discussed for the magnetic white dwarfs PG 1031+234 (Latter et al. 1987) and RE 0317-853 (Vennes et al. 2003). The origin of such a spot-like feature, is however unclear although star spots have been found on the possible progenitors of magnetic white dwarfs, the Ap stars, where the spots are connected to abundance anomalies and can be detected via Doppler imaging (Khokhlova et al. 1986; Rice \& Wehlau 1994).

An extremely homogeneous field was also found in phaseresolved low-state spectra of the polar MR Ser. Schwope et al. (1993) revealed a strongly decentered dipolar $\left(z_{\text {off }} \approx 0.3\right.$ stellar radii).

However, it must be pointed out that in Ap stars, which also have fossil magnetic fields, low order multipole expansions describe statistical aspects of overall field geometry, but do not provide a realistic representation of the actual distribution of field vectors over the surface; the latter would be more constrained by observations of circular polarization. Therefore, our solution only means that the field modulus is quite uniform and large over the visible hemisphere of the star. The relatively small region with a higher field modulus does not strongly contribute to the strength of the line features.

In the framework of multipole exansions Muslimov et al. (1995) have shown that a weak quadrupole (or octupole, etc.) 
component on the surface magnetic field of a white dwarf may survive the dipole component und specific initial conditions: particularly the evolution of the quadrupole mode is very sensitive (via Hall effect) to the presence of internal toroidal field. For a $0.6 M_{\odot}$ white dwarf with a toroidal fossil magnetic field of strength $<10^{9} \mathrm{G}$ the dipole component declines by a factor of three in $10^{9} \mathrm{yr}$, while the quadrupole component is practically unaffected. Without an internal toroidal field the dipole component still declines by a factor of three but the quadrupole component is a factor of six smaller after $10 \mathrm{Gyr}$. This is much longer than the cooling age of a $12000 \mathrm{~K}$ white dwarf, which well under $1 \mathrm{Gyr}$ for $0.6 M_{\odot}$ and larger than $3 \mathrm{Gyr}$ only for masses larger than $1.2 M_{\odot}$.

Whether spot-like structures could also survive for the long cooling time of white dwarfs has to be investigated theoretically.

If a magnetic spot is indeed present a strong temporal variation of the spectrum and particularly the polarization is expected, if the star is rotating. However, no significant differences could be found between the spectra taken in September, 1998 and November, 1998, respectively. This may be an indication for only slow rotation as known in the case of Grw $+70^{\circ} 8247$ where a rotational period of decades or centuries is possible (Schmidt \& Norsworthy 1991; Friedrich \& Jordan 2001). Therefore, a more detailed analysis and a decision whether a spot is present has to wait until time resolved spectro-polarimetry of this object becomes available.

Acknowledgements. We thank the referee John Landstreet for his very valuable comments particularly on the situation in Ap stars. Work on magnetic white dwarfs in Tübingen is supported by the DLR grant 50 OR 0201.

\section{References}

Angel, J. P. R., Liebert, J., \& Stockman, H. S. 1985, ApJ, 292, 260 Euchner, F., Jordan, S., Beuermann, K., et al. 2002, A\&A, 390, 633 Carroll, D. L. 2001, http: //cuaerospace.com/carroll/ga.html Chabrier, G., Brassard, P., Fontaine, G., \& Saumon, D. 2000, ApJ, 543, 216

Friedrich, S., \& Jordan, S. 2001, A\&A, 367, 577

Greenstein, J. L. 1984, ApJ, 281, L47

Harris, H. C., Liebert, J., Kleinman, S. J., et al. 2003, AJ, 126, 1023

Jordan, S. 1989, in White Dwarfs, ed. G. Wegner (New York: Springer-Verlag), Lecture Notes in Physics, 328, 333

Jordan, S. 1992, A\&A, 265, 570

Jacobs, J. A. 1987, Geomagnetism, Vol. 1 (London: Academic Press Limited)

Kemp, J. C. 1970, ApJ, 162, 169

Khokhlova, V. C., Rice, J. B., \& Wehlau, W. H. 1986, ApJ, 307, 768

Minkowski, R. 1938, Ann. Rept. Div. Mt. Wilson Obs., 38

Latter, W. B., Schmidt, G. D., \& Green, R. F. 1987, ApJ, 320, 308

Muslimov, A. G., Van Horn, H. M., \& Wood, M. A. 1995, ApJ, 442, 758

Praddande, H. C. 1972, Phys. Rev. A, 6, 1321

Reimers, D., \& Wisotzki, L. 1997, The Messenger, 88, 14

Rice, J. B., \& Wehlau, W. H. 1994, A\&A, 291, 825

Schmidt, M., \& Green, R. F. 1983, ApJ, 269, 352

Schmidt, G. D., \& Norsworthy, J. E. 1991, ApJ, 366, 270

Schwope, A. D., Beuermann, K., Jordan, S., \& Thomas, H.-C. 1993, A\&A, 278, 487

Stevenson, C. C. 1994, MNRAS, 267, 904

Vennes, S., Schmidt, G. D., Ferrario, L., Christian, D. J., Wickramasinghe, D. T., \& Kawka, A. 2003, ApJ, 593, 1040

Wickramasinghe, D. T., \& Ferrario, L. 1988, ApJ, 327, 222

Wisotzki, L., Köhler, T., Groote, D., \& Reimers, D. 1996, A\&AS, 115, 227 